\title{
Characterization and control of a bottleneck-induced traffic-jam transition for self-propelled particles in a track
}

\author{
Thomas Barois, Jean-François Boudet, Nicolas Lanchon, Juho S. Lintuvuori, and Hamid Kellay \\ Université Bordeaux, CNRS, LOMA, UMR 5798, F-33405 Talence, France
}

(Received 21 November 2018; revised manuscript received 22 February 2019; published 13 May 2019)

\begin{abstract}
A collection of self-propelled elongated particles is circulating in a circular track. Due to the presence of a bottleneck, the flow transits to a congested state for a sufficient number of particles, even if the whole track is not saturated. Both experiments and simulations are used to identify the transition toward congestion. An intermediate regime of coexistence is characterized by intermittency between a free flow state and a jammed state. The range of the coexistence region is found to depend explicitly on fluctuating quantities such as the distribution of the escape times from a jam and the headway time distribution between free particles. Optimization strategies, such as the "slower is faster" effect, are tested in experiments and simulations, and an increase in the traffic performances is reported.
\end{abstract}

DOI: 10.1103/PhysRevE.99.052605

\section{INTRODUCTION}

Assemblies of self-propelled particles are model systems to mimic the collective behaviors observed in complex active systems [1-4]. The approach is to reduce the complexity of the individual elements in order to reveal the physical mechanisms at the origin of the emergence of a collective dynamic. This work focuses on the emergence of traffic jams as a collective effect of particle ensembles with physical interactions.

The concept of jamming is encountered in a wide variety of situations, such as road [5-7] and internet [8] traffic, escape from confined environments [9-11], or granular flows [12-15]. The typical physical approaches are based on models in which the flow dynamics depends on the local density. This is the case for continuous models derived from fluid dynamics $[16,17]$ or hopping models using cellular automata $[18,19]$.

A variety of model experiments have been proposed to explore the traffic of self-propelled elements in roadlike geometries. For noncontact interactions mediated by a diffusing surfactant [20] or light sensing [21], the traffic flow limitation is associated with the saturation of the track. Self-propelled particles with contact interactions have been used to explore either a free regime of traffic [22] or the congested regime obtained with particles in a hopperlike constriction [23].

In this work, we explore the transition between free flow and a jammed state for a set of self-propelled elements. Here, the emergence of jams is not related to saturation of the whole track but to the presence of a bottleneck. The model particles used in the experiments are autonomous robots with contact interactions, and they are constrained to move in a track with a localized reduction in width. A transition from a free-flowing to a congested state is observed when the number of particles is increased. First we characterize the transition and show that it differs from what would be observed for jams occurring due to the track saturation mechanism. Second, we investigate some strategies to shift the onset of congestion and we observe either a decrease or an increase in the overall traffic performances for the particle ensemble.

To probe the traffic performances of active particles in a model geometry, the relation between the average flux and the number of particles in the track is used. This representation is analogous to the so-called fundamental diagram [24-26] usually encountered in traffic engineering. The fundamental diagram represents the vehicle flux at a counting point as a function of the vehicle density. At low densities, the flux increases linearly with the vehicle density. The associated slope is the free velocity that is weakly affected by the density variations in this regime. For higher densities, the appearance of traffic jams drastically modifies the flow, and the overall traffic decreases with the increase in the number of vehicles.

The triggering of jams is related to a wide variety of causes, such as obstacles and lane reduction [27-29], road crossings [30], or density fluctuations without bottlenecks [24,31]. However, the fundamental diagram systematically shows the increasing and decreasing regimes discussed above. If the increasing regime is expected, the regime with a decrease of the traffic with an increase of the number of vehicle is counterintuitive. The physical systems with self-propelled particles mentioned before [18-21] capture such a decrease in the case of track saturation.

Improving the traffic performances close to track saturation is possible, but it requires significant modifications of the particles' properties, such as their sizes or their mean velocities. Notably, the reduction of velocity is known to improve the traffic performances near congestion according to the "slower is faster" effect reported in model systems and road or pedestrian traffic measurements [32-38]. Here, we show that it is possible to go beyond the "slower is faster" effect in our simple system: a regulation strategy is tested in the simulations where the average properties of the particles are not modified. Instead, particles are locally slowed down before the bottleneck, leading to a more uniform particle distribution and an overall increase in the flow behavior. 


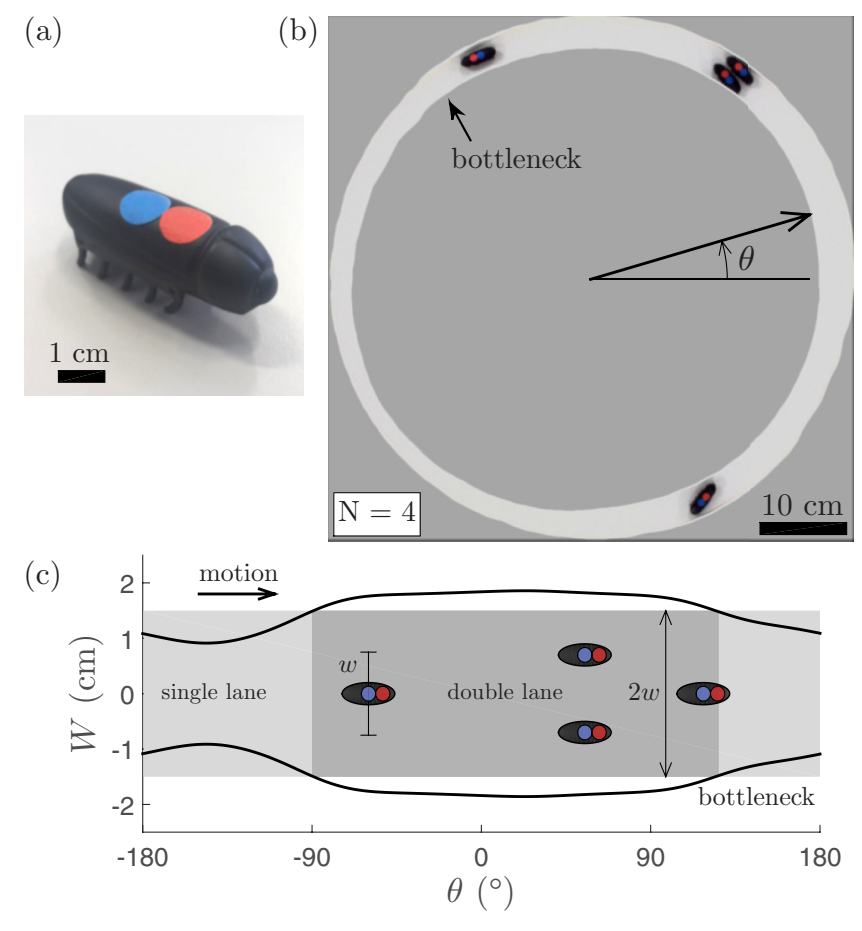

FIG. 1. (a) Electromechanical rodlike robots used in the experiments. (b) Snapshot of the traffic experiment for $N=4$ selfpropelled particles turning anticlockwise in the track. The location of a particle in the track is given by the angle $\theta$. (c) Width $W$ of the track (solid line) as a function of the angular position $\theta$.

\section{EXPERIMENTAL SETUP}

Two types of self-propelled electromechanical particles are used in this work: dry granular particles (self-propelled rodlike robots) and particles moving close to an air-water interface in a shallow tank (fishlike robots). The main emphasis of this work concerns the dry particles, while the swimming particles are used to test how different escape time statistics affect the jam dynamics.

The dry granular particles are electromechanical robots, nearly ellipsoidal, with an aspect ratio close to 3 (length $\ell=4.4 \mathrm{~cm}$, width $w=1.5 \mathrm{~cm}$ ) [Fig. 1(a)]. The robots have a battery and a vibrating element that leads to a unidirectional propulsion in the tail-to-head direction [39-41]. The randomness of the shocks due to the vibration results in an effective diffusion of the orientation of the robot without confinement.

The swimming fish robots have dimensions of $7.3 \mathrm{~cm}$ in length, $3.2 \mathrm{~cm}$ in height, and $1.7 \mathrm{~cm}$ in width. They are propelled by the flapping of a caudal fin (operated by a set of two batteries and an electromagnetic motor) and move at velocities between 10 and $15 \mathrm{~cm} \mathrm{~s}^{-1}$. The track was immersed in a water pool roughly $5 \mathrm{~cm}$ in height, and the fish robot motion is nearly bidimensional.

The experiments with the dry granular particles (rodlike) are conducted in a circular track of length $L=182 \mathrm{~cm}$ with a varying width. The revolution period for a single particle is $T_{t}=5.5 \mathrm{~s}$ and the maximal capacity of the track is 78 particles. Figure 1(b) shows a snapshot of the traffic experiment with four self-propelled vehicles in motion. The width of the track $W(\theta)$ is presented in Fig. 1(c). $W(\theta)$ does not exceed
$4 \mathrm{~cm}$, and the particles cannot reverse their direction. A wider track would allow the particles to change their orientation and possibly to form clusters mediated by the walls [40-44]. The track has a bottleneck similar to a 2-to-1 lane reduction on a road: on half of the track, overtaking is possible with $W(\theta)>2 w$, and on the other half only one robot can go through because $w<W(\theta)<2 w$.

The average traveling velocity of the particles is $v_{T}=33$ $\mathrm{cm} \mathrm{s}^{-1}$. This average velocity varies by about $2 \mathrm{~cm} \mathrm{~s}^{-1}$ between two particles. For a given particle, the velocity fluctuates over time with a standard deviation of $3 \mathrm{~cm} \mathrm{~s}^{-1}$. Here, the velocity fluctuations are sufficiently large so that the particles can catch up with each other in the double lane sector.

\section{EXPERIMENTAL RESULTS}

\section{A. Transition to congestion}

A set of movies is recorded for a varying number $N$ of particles in the track (see the Supplemental Material [45]). For a few particles, as in Fig. 1(a) with $N=4$, the flow is free. At higher numbers, a clogging of particles is observed at the bottleneck, as shown in Fig. 2(a).

The relation between the flux $Q$ and the total number of particles $N$ in the track is represented in Fig. 2(b) with filled circles. The flux is measured by counting the particles crossing the position $\theta=-90^{\circ}$ with a fixed $N$ in the track during the acquisition time. A free regime is observed at low $N$ where $Q$ varies linearly with $N$. For high values of $N>20$, a congested regime is observed and the flux is strongly reduced in comparison to an extrapolated free regime. There is a finite flux in this regime since the particles may escape from the jam, but there is permanently a particle population blocked at the bottleneck.

A transition region is observed for the range $9 \leqslant N \leqslant 18$, where, for a fixed $N$, the flow switches spontaneously between the free and the congested regimes. The instantaneous flow is presented with the angular trajectories in Fig. 2(c) for $N=5,15$, and 22 particles. For $N=5$, the angle $\theta$ increases linearly with time for all the trajectories indicating a freeflowing regime. For $N=15$, this angular dynamics shows both linear regimes, indicating free flow, as well as blocked regimes where the angle varies little with time. The particles in the jam form a horizontal band at the bottleneck below $\theta=126^{\circ}$. For $N=22$, this horizontal band is persistent in the trajectory diagram, which means that the state is exclusively jammed.

The instantaneous velocities of the particles are computed from the recorded trajectories. Figure 3 shows a representation of the particle velocity distributions depending on the number of particles in the track $N$. At low $N$, the velocity distribution is monomodal and centered on the typical velocity $v_{T}=$ $33 \mathrm{~cm} \mathrm{~s}^{-1}$ of the particles freely moving in the track. Approaching $N=10$, the velocity distribution is slightly shifted to a lower value, typically $30 \mathrm{~cm} \mathrm{~s}^{-1}$, which corresponds to the velocity of the slowest particles in the track. This velocity reduction is consistent with the formation of moving clusters [22] with the slowest particles ahead. The velocity distribution becomes bimodal in the coexistence region $9 \leqslant N \leqslant 18$, and the mode related to the particles in the 
(a)

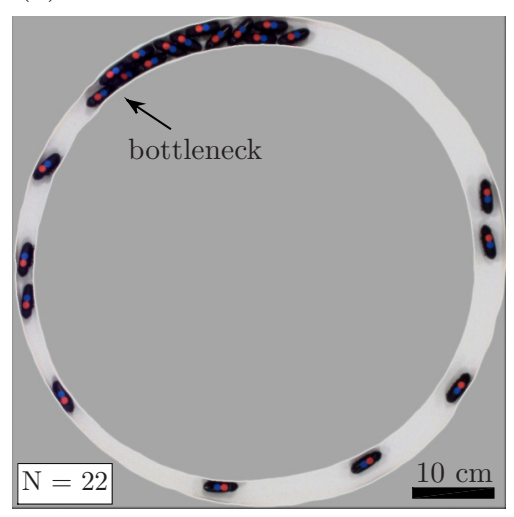

(b)

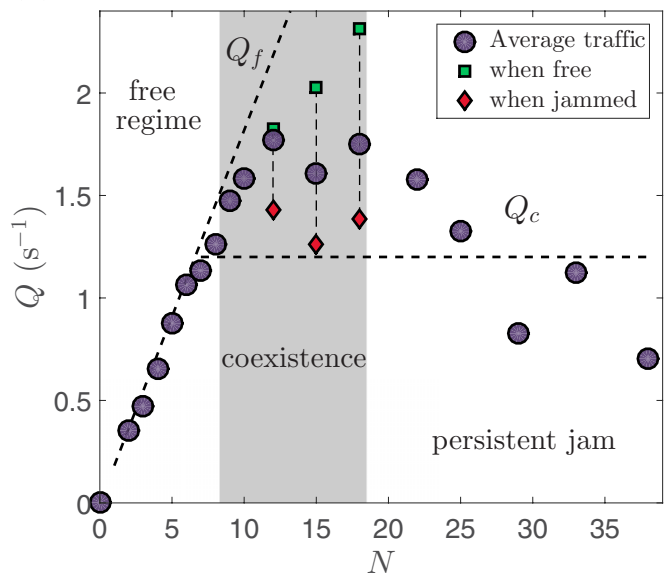

(c)

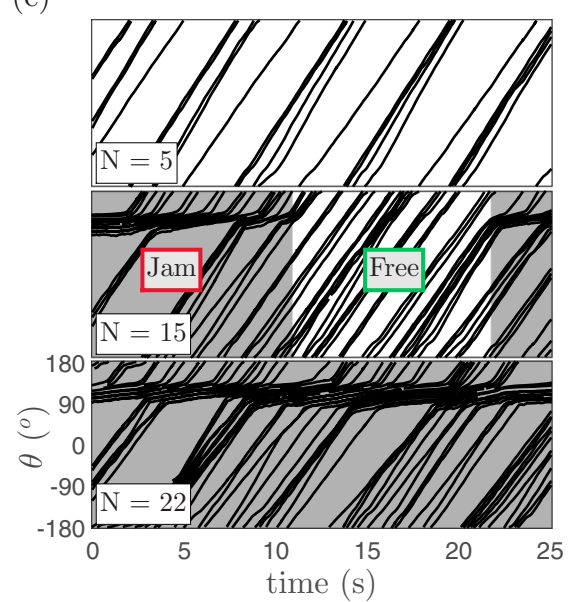

FIG. 2. (a) Snapshot of the traffic experiment for $N=22$ self-propelled particles. At the bottleneck, 13 particles are packed in a slowly flowing jam. (b) Particle flux $Q$ as a function of the total number of particles $N$ (filled circles). For $9 \leqslant N \leqslant 18$, there is a switching between the jammed and the free regimes over time at fixed $N$. Outside this range, the regime is exclusively free or congested. In the coexistence region, the flux is computed over the time period for which the flow state is either free (squares) or jammed (diamonds). The dashed lines are for the free flux $Q_{f}=N / T_{t}$ and the congested flux $Q_{c}=1.2 \mathrm{~s}^{-1}$. (c) Angular trajectories $\theta(t)$ of the particles for $N=5,15$, and 22 corresponding to the free, the coexisting, and the jammed regimes, respectively. The snapshot (a) corresponds to the timeline $7.8 \mathrm{~s}$ in the trajectory for $N=22$.

jam has a typical velocity of $5 \mathrm{~cm} \mathrm{~s}^{-1}$. The threshold value of $15 \mathrm{~cm} \mathrm{~s}^{-1}$ is selected to discriminate between particles belonging to the free mode with high velocities or the jammed mode at low velocities. This threshold is about half of the free velocity. At higher $N$, the velocity distribution is dominated by the contribution of the particles in the jam even if a small proportion of the particles still escapes from the jam.

For each recording at constant $N$, the flux is computed separately over the time period for which the flow is either free or jammed. To do so, the existence of a jam is deduced from the instantaneous particles velocities: if at least $N-1$ particles

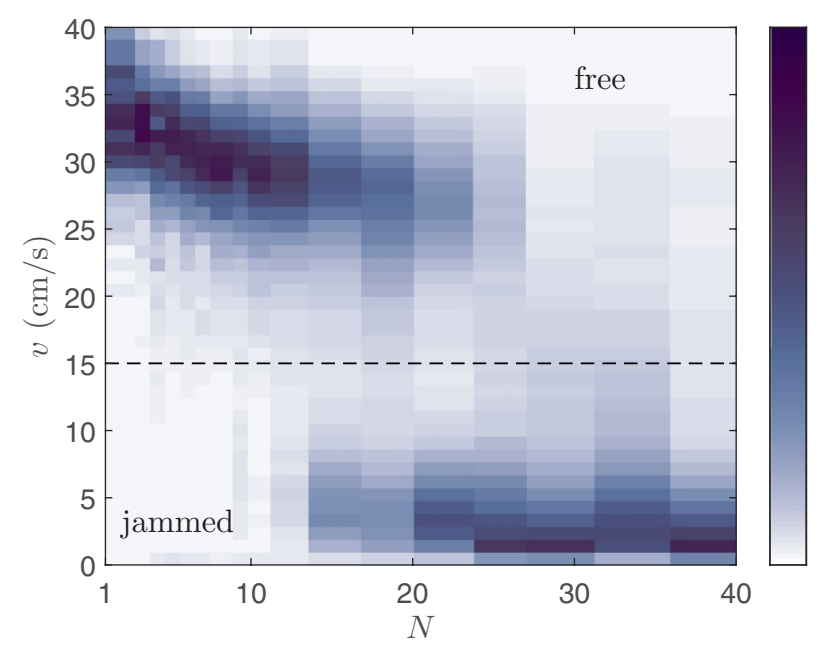

FIG. 3. Velocity map of the self-propelled particles in the traffic experiment with the dry granular robots. A linear color scaling is used to represent the probability to have a particle having a velocity $v$ with a total number of $N$ particles in the track. The dashed line $v=15 \mathrm{~cm} \mathrm{~s}^{-1}$ indicates the threshold value between a particle in a jam and a free particle. are moving faster than the threshold velocity of $15 \mathrm{~cm} \mathrm{~s}^{-1}$, the flow state is considered to be free. The angular trajectories for $N=15$ show two spontaneous transitions between the jammed and the free states. A decomposition in two branches is obtained in the coexistence region as presented in Fig. 2(b), where $Q_{f}$ and $Q_{c}$ are the free and the congested branches.

\section{B. Two-population stationary model}

A first analysis of the flow state is proposed with stationary arguments. The traffic of the particles is described by two populations where $n_{J}(t)$ is the number of particles in the jam and $N-n_{J}(t)$ is the number of free particles in the track. Without a jam $n_{J}(t)=0$, the flux is given by $Q_{f}=N / T_{t}$. If there is a jam $n_{J}(t) \geqslant 2$, the particles may escape from the jam with a distribution of escape times. For a persistent jam, the average flux is given by the inverse of the average escape time $Q_{c}=1 / \overline{T_{e}}$. An average escape time $\overline{T_{e}}=0.82 \mathrm{~s}$ is obtained from the measurement of the escape time distribution. The associated flux $1 / \overline{T_{e}}=1.2 \mathrm{~s}^{-1}$ is represented by the horizontal dashed line $Q_{c}$ in Fig. 2(b). It gives a satisfying description for the jammed branch near the coexistence region. In the persistent jam region, $Q$ tends to decrease with increasing $N$. A more accurate description of the escape dynamics could be proposed with $1 / \overline{T_{e}}$ depending on $N$. Because this study is focused on the transition region, only a simplified approach is considered where the average escape time $\overline{T_{e}}$ is independent of $N$.

The relation between the average number of particles in the jam $\overline{n_{J}}$ and $N$ can be established in the stationary limit. For a jam with an average $\overline{n_{J}}$ particles, each particle spends a time $\overline{n_{J}} \overline{T_{e}}$ in the jam and a time $T_{t}$ in the track giving $\overline{n_{J}}=$ $N \times \overline{n_{J}} \overline{T_{e}} /\left(\overline{n_{J}} \overline{T_{e}}+T_{t}\right)$ and consequently

$$
\overline{n_{J}}=N-\frac{T_{t}}{\overline{T_{e}}} .
$$




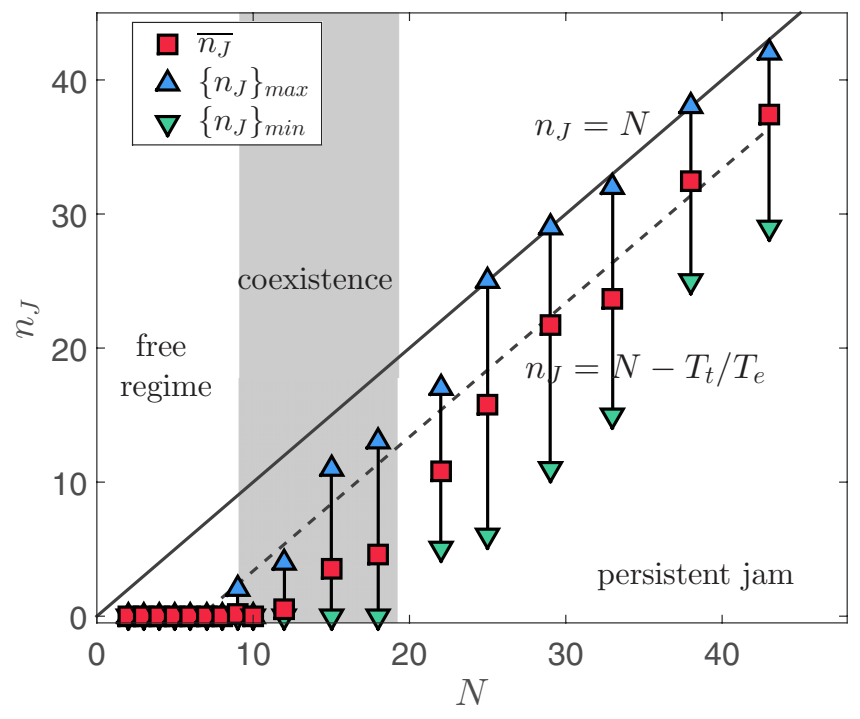

FIG. 4. Number of jammed particles $n_{J}$ as a function of the total number of particles $N$ in the track. The square symbols correspond to the average value $\overline{n_{J}}=\left\langle n_{J}(t)\right\rangle_{t}$ during an acquisition with fixed $N$. The maximum value of $n_{J}(t)$ during an acquisition is represented by triangles pointing upward and downward, respectively, for the minimum value. The solid line is the upper bound $n_{J}=N$ for which all the particles are in the jam. The dashed line is the relation (1). In the coexistence region, $\overline{n_{J}}>0$ and $\left\{n_{J}\right\}_{\min }=0$.

The ratio $T_{t} / \overline{T_{e}}=N-\overline{n_{J}}$ fixes the average number of free particles when a persistent jam is formed.

The beginning of the coexistence region is consistent with $N-\overline{n_{J}}=T_{t} / \overline{T_{e}}=6.6$ with a threshold $\overline{n_{J}}=2$ clogged particles. Below $N=9$, the jams that might eventually form vanish rapidly as not enough particles feed the jam since most of their time has to be spent in the free-flowing part of the track.

\section{Role of the jam size fluctuations}

The analysis of the traffic by stationary flow states describes both the free and the congested regimes with $Q_{f}=$ $N / T_{t}$ and $Q_{c}=1 / T_{e}$, respectively. A critical number $N_{c}=$ $T_{t} / \overline{T_{e}}$ corresponding to a crossover for the two regimes $Q_{f}=$ $Q_{c}$ is defined, but the details of the transition are not predicted.

Figure 4 presents the number of jammed particles $n_{J}$ as a function of the total number of particles $N$ in the track. In the free regime $N<9, n_{J}=0$. In the jammed regime, the average number of jammed particles $\overline{n_{J}}$ is consistent with Eq. (1). The instantaneous number of jammed particles $n_{J}(t)$ fluctuates between a lower value $\left\{n_{J}\right\}_{\min }>0$ and an upper value $\left\{n_{J}\right\}_{\max } \sim N$ corresponding to all the particles in a jam. In the range $9 \leqslant N \leqslant 18, \overline{n_{J}}$ is nonzero, and jams appear after a finite time. However, the jam state is not persistent because $\left\{n_{J}\right\}_{\min }=0$, which means that the fluctuations of jam size are large enough to result in a complete dissolution of jams.

To show how the jam size fluctuations may modify the traffic diagram, a similar traffic experiment was performed with the self-propelled particles mimicking swimming fishes. The artificial fishes are confined in an annular tank with a
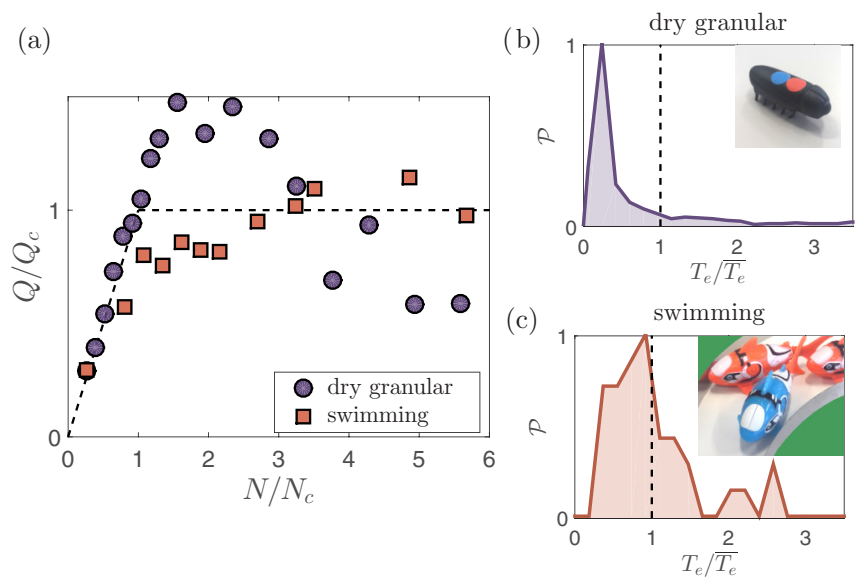

FIG. 5. (a) Particles flux as a function of the number of particles for the two experiments with the two types of self-propelled particles circulating in a track with a bottleneck. $Q$ and $N$ are normalized with $Q_{c}=1.2 \mathrm{~s}^{-1}$ and $N_{c}=7.7$ for the dry granular and $Q_{c}=0.19 \mathrm{~s}^{-1}$ and $N_{c}=3.7$ for the artificial fishes. (b) Escape time distribution from a jam with dry self-propelled particles. Average time $\overline{T_{e}}=$ $0.83 \mathrm{~s}$, standard deviation $\delta T_{e}=1.8 \mathrm{~s}$. (c) Escape time distribution from a jam with swimming artificial fishes. $\overline{T_{e}}=5.4 \mathrm{~s}, \delta T_{e}=3.1 \mathrm{~s}$.

bottleneck. The track profile is similar to the track profile used for the dry granular robots presented in Fig. 1. The flux $Q$ of the dry granular and the swimming self-propelled particles is presented in Fig. 5(a) as a function of the total number of particles $N$. The representation of the flux is in normalized units with $Q / Q_{c}=1$ in the jammed regime. $N_{c}$ is such that the slope in the free regime is unity. In the case of the swimming particles, the two regimes, free and congested, are clearly identified, but, contrary to the dry granular particles, there are almost no data points above the congested branch $Q / Q_{c}=1$.

The swimming particles were chosen because they have a dynamics of escape from jams that differs from the escape dynamics of the dry particles. Figure 5 presents the escape time distributions for the dry granular particles (b) and the artificial fish robots (c). For both particles, an average escape time $\overline{T_{e}}$ is defined and it is used to characterize the jammed regime $Q / Q_{c}=1$ in the plot (a) with $Q_{c}=1 / \overline{T_{e}}$. For the artificial fish, $\overline{T_{e}}=5.4 \mathrm{~s}$ and the maximum of the distribution is relatively close to $\overline{T_{e}}$. In the case of the dry granular particles, the maximum of the distribution is located at a time typically four times smaller than the average escape time $\overline{T_{e}}=$ $0.83 \mathrm{~s}$. The relative amplitude of the fluctuations is computed as the ratio between the standard deviation $\delta T_{e}$ and the average escape time $\overline{T_{e}}$. For the artificial fish, $\delta T_{e} / \overline{T_{e}}=0.58$ while for the dry granular, $\delta T_{e} / \overline{T_{e}}=2.16$. Consequently, the fluctuations of escape time are much larger for the dry granular particles, which means that $n_{J}(t)$ fluctuates sufficiently to dissolve almost any jam in the region $1<N / N_{c}<2$. In the limit of no fluctuations and large $N_{c}$, the flow regime would follow the two regimes $Q_{f}$ and $Q_{c}$ represented by a dashed line in Fig. 5(a) with a sharp transition at $N=N_{c}$. While the artificial fishes with smaller fluctuations in escape time statistics follow this trend, the dry particles with large fluctuations in escape times do not. This points to the importance of 


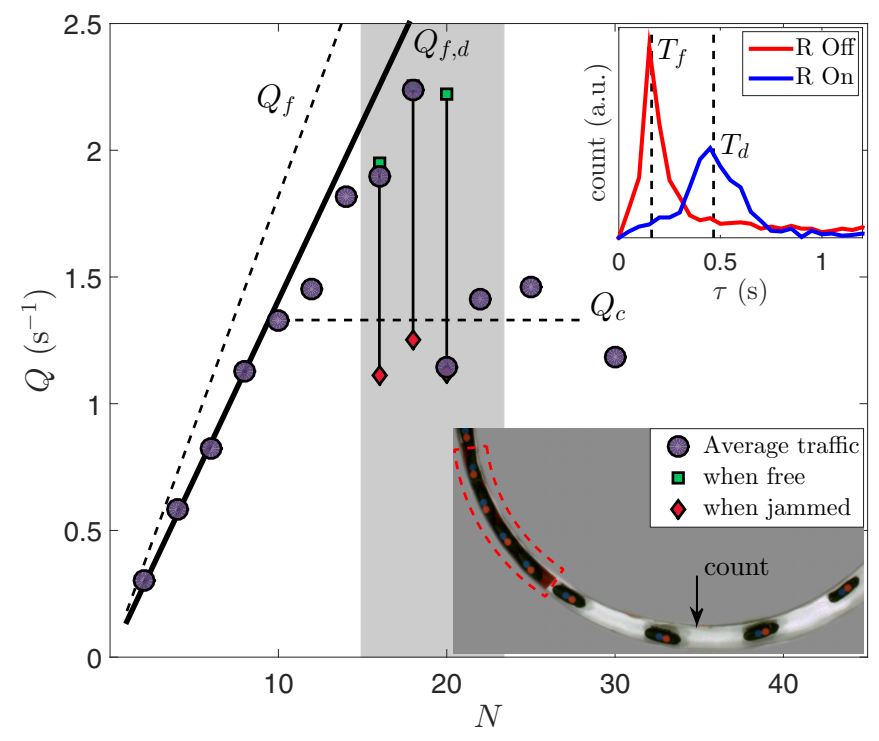

FIG. 6. Vehicle flux $Q$ as a function of the number of particles $N$ with a damper. The inserted image shows the location of the damper that is a soft sheet positioned in the track. The dashed lines are the same as in Fig. 2(b). The solid line is for the free branch with damper $Q_{f, d}=N / T_{t}$ with $T_{t}=7.1 \mathrm{~s}$. The inserted plot shows the time delay probability between two particles following each other at the counting point $\theta=-90^{\circ}$. Without the damper, the time delay is maximal for particles following each other with $T_{f}=\ell / v_{T}$. With the damper, the maximum is shifted at $T_{d}=\ell /\left(v_{T}-v_{D}\right)$.

escape time statistics in setting the characteristics of the traffic diagram.

\section{Regulation by a damper}

A regulation strategy is tested with a modification of the particle dynamics in the track. The regulation mechanism implemented relies on the concept of "slower is faster."

To reduce the velocity of the particles, a damper, i.e., a thin layer of soft plastic, is positioned in the track in the single lane sector. This soft sheet covers the track width over a length of $L_{D}=19.5 \mathrm{~cm}$ between $\theta=-170^{\circ}$ and $-133^{\circ}$. A dashed line is used in the inserted image in Fig. 6 to signal the location of the soft sheet. The sheet is not clearly visible in the image because it is covered by some particles. The thin sheet acts as a damper: the particle self-propulsion is less efficient on the damper because of its softness, and the average velocity is reduced to $v_{D}=9 \mathrm{~cm} \mathrm{~s}^{-1}$.

The traffic relation with the damper is shown in Fig. 6. In the free regime at low $N$, the flux is reduced compared to the first experiment without a damper, simply because the flux is inversely proportional to the revolution period. The coexistence between jammed and free flow is still observed for an intermediate number of particles. However, the region of the free flow is increased up to $N=18$ particles. For high numbers $N \geqslant 25$, the flux is comparable to what is measured without regulation.

The presence of the damper increases the revolution period to $T_{t}=7.1 \mathrm{~s}$. The traffic improvement by the regulation with the damper is effective for intermediate $N$ in the coexistence region. The shift of the coexistence region toward higher $N$ is consistent with the presence of the damper and the revolution period $T_{t}$ increase. In the stationary model described before, the number of particles in the jam $n_{J}$ is expected to decrease with an increase of the revolution period $T_{t}$ according to Eq. (1).

The maximal flux with the damper is $Q=2.2 \mathrm{~s}^{-1}$ for $N=18$. Without a damper the maximal flux is $Q=1.8 \mathrm{~s}^{-1}$ for $N=12$. The effect of the velocity reduction alone should not result in an increase of the maximal flux. To a first approximation, the velocity reduction and the associated increase of $T_{t}$ should be equivalent to an increase of the track length for an unmodified velocity. Since the vehicle density goes as the inverse of the track length, the velocity reduction should be equivalent to a density reduction and a shift of the flux relation with a rescaling for $N$ and without modification for $Q$. This scaling argument is supported by the measurements on road traffic [38] in which the flow diagram appears to be a function of the occupancy divided by the inverse of the speed limits. Accordingly, the maximal vehicle flow reported is independent of the speed limitations.

The increase of the maximal flux is related to the fact that the damper is not only increasing the revolution period but it also modifies the spacing between the particles. The inserted plot in Fig. 6 shows the headway time distribution at the counting point $\theta=-90^{\circ}$, just after the damper. Without damper regulation ( $\mathrm{R}$ Off), the headway time probability is maximal for $T_{f}=0.15 \mathrm{~s}$, which corresponds to the typical headway time $\ell / v_{T}=0.13 \mathrm{~s}$ for two particles following at contact distance. With the damper ( $\mathrm{R} \mathrm{On}$ ), the maximum of the headway time probability is shifted to $T_{d}=0.45 \mathrm{~s}$. Converted to a spacing distance $T_{d} v_{T}-\ell$, it corresponds to a typical separation between following particles of two particle lengths. The consequence of this separation is to decrease the encounter probability at the bottleneck and then to decrease the clogging probability. This points to the importance of headway time statistics on setting the details of traffic diagrams.

\section{DYNAMICAL MODEL}

In the previous section, a stationary analysis has been proposed based on the existence of an average revolution time $T_{t}$ and an average escape time $T_{e}$. This analysis is not sufficient to describe the details of the transition to congestion because the dynamics of the particles and notably their fluctuations have to be considered. The object of this section is to introduce a dynamical model that captures the physics of the transition from free flow to congestion and to pinpoint the role of the fluctuations.

\section{A. Lattice model}

The dynamics of the particles and the transition to congestion are addressed by a lattice model in which both time and space are discrete quantities. The particles are assimilated to cellular automata moving in a discrete track with periodic boundaries composed of a single lane with 50 cells followed by a double lane with $50 \times 2$ cells. The particles obey the exclusion rule that states that a particle does not move to 


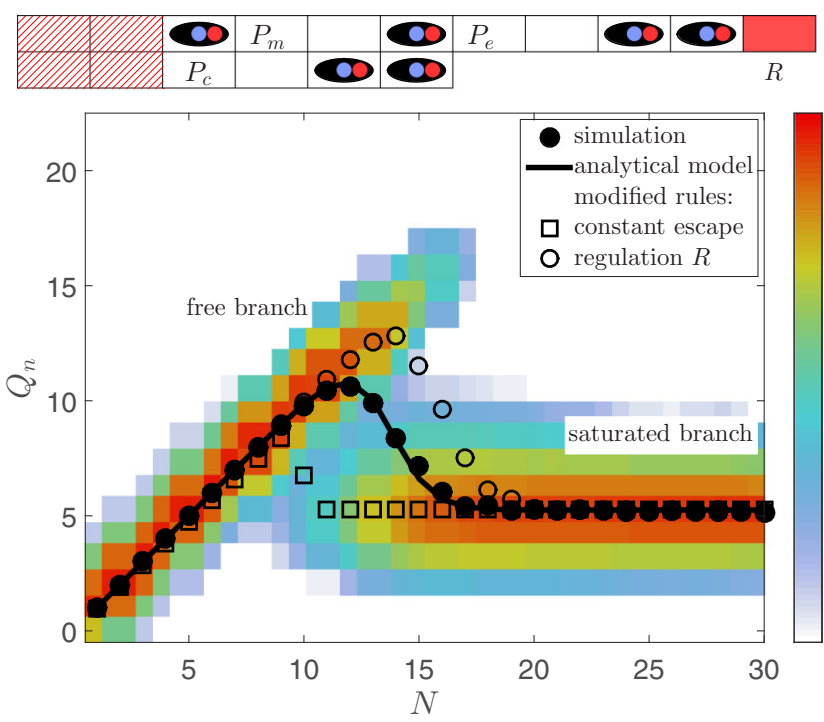

FIG. 7. Simulated flow diagrams $Q_{n}=f(N)$ for particles in a periodic discrete track (represented in the schematic). The probabilities for the particles to move at each time steps are $P_{m}=0.95$, $P_{c}=0.1$, and $P_{e}=0.05$ (see the text). The simulation represented by filled circles is for the set of probabilities $\left\{P_{m}, P_{c}, P_{e}\right\}$ with no further rules. The square symbol is the traffic relation when the escape time distribution is a narrow Gaussian corresponding to a nearly constant escape time $\tau \approx P_{e}{ }^{-1}$. The traffic relation with open circles is obtained with the set $\left\{P_{m}, P_{c}, P_{e}\right\}$ plus a regulation of the particle distances. The regulation rule is for the last cell $R$ of the track where a particle is allowed to move forward if the next $n=5$ cells are empty ( $n=2$ in the schematics). The flux is in normalized units $Q_{n}=Q N_{c} / P_{m}$, where $N_{c}=100$ is the cell length $\left(N_{c}=10\right.$ in the schematics). The density map relates to the simulation presented by filled circles, and it gives the probability to find a flux $Q_{n}$ for $N$ particles in the track.

an occupied cell. The dynamics of the particles follows three rules.

First, for each time step the particles have a probability $P_{m}=0.95$ to move forward. $P_{m}$ is not exactly equal to unity in order to induce some fluctuations in the trajectories and allow interactions between particles via the exclusion rule.

Second, in the double-lane sector, both lanes are explored via a probability $P_{c}=0.1$ to change lanes at each time step. The two processes of moving forward and changing lanes are independent.

The last pair of cells in the double-lane sector is mimicking the bottleneck via a third rule. If there is only one particle on the last pair of cells, the particle dynamics is the same as before. If two particles occupy the last pair of cells, the particle in one of the lanes has a probability $P_{e}=0.05$ to move forward.

\section{B. Lattice model solution}

\section{Numerical solution}

The results of the simulation of the lattice model previously described are represented by filled circles in Fig. 7 .

The simulations are carried out for $10^{6}$ time steps with $N=1, \ldots, 30$ particles. $N$ is varied in a regime below the track saturation that would occur for $N$ approaching the 150 cells of the track. The trajectories of the cellular automata are processed to define an instantaneous flux per particle $1 / T$, where $T$ is the number of time steps to cross the full track. This flux per particle is in normalized units $T(1) / T$ with $T(1)=N_{c} / P_{m}$, the mean revolution time for a single particle, where $N_{c}=100$ is the linear number of cells. The total normalized flux $Q_{n}$ is the sum of $T(1) / T$ over the $N$ particles. The probability map in Fig. 7 represents in a logarithmic color scale the total flux distribution and its average value with filled circles. The statistics of the flux shows two branches associated with $Q_{n, f}=N$ for the free regime and $Q_{n, c}=$ $P_{e} N_{c} / P_{m}$ for the congested regime. Coexistence between the two regimes is observed, typically for $10 \leqslant N \leqslant 18$, where the flow state spontaneously switches between the free and the congested regime as observed in experiments.

An additional set of simulations, not presented in the figure, is performed to investigate the influence of the moving probability $P_{m}$. For $P_{m}=1$, the flow regime is exclusively free, simply because the particle spacing is independent of time and there is no possible jam nucleation. In the range $0.90 \leqslant P_{m} \leqslant 0.96$, the jam nucleation is possible and almost independent of the value of $P_{m}$, with a relative variation for $Q_{n}$ of $5 \%$ at most. This is because the motion fluctuations $1-$ $P_{m}=5 \%$ are large enough so that the particles randomly catch up with each other in the double-cell sector and eventually arrive simultaneously at the bottleneck. With $P_{m}=0.95$, the particle dispersion is typically 1.5 cells after the 50 cells of the double-lane sector. The transition to the correlated motion is typically for $P_{m}=0.98$, for which the particle dispersion after 50 cells is 1.0 .

Similarly, a transition to a single file motion in the doublelane sector would be obtained for $P_{c}<0.02$, meaning that $P_{c}$ would be too small for the particles to explore the second lane.

\section{Analytical solution}

An analytical model is proposed to account for the flux relation observed in the simulation presented with filled circles in Fig. 7. For a fixed $N$, the average flux depends on (i) the typical duration of a free state and (ii) the typical duration of a jammed state. The solution is established in the limit of particles moving without synchronization in the double-lane sector $\left(P_{m}<0.98\right)$ and exploring both lanes $\left(P_{c}>0.02\right)$.

For (i), with an initial free state, a jam forms when two particles simultaneously occupy the last two sites of the double lane. Assuming that all the configurations are equivalent, the probability $P_{j}$ to form a jam at a given time step is $P_{j}=[N \times$ $(N-1)] /\left[N_{T} \times\left(N_{T}-1\right)\right]$ with $N_{T}=150$ the total number of cells. The average duration of the free state is simply $P_{j}^{-1}$.

For (ii), and with an initial congested state, the jam is effectively dissolved when the number of escape events is at least $N$ during a mean revolution time. $N_{R} \approx 105$ represents the number of escape attempts during $N_{c} / P_{m}$ time steps after which an escaped particle returns to the jam. The probability to have $N_{i}$ particles escaping at a rate $P_{e}$ with $N_{R}$ attempts is given by the binomial relation $\mathcal{B}\left(N_{i}\right)=\mathcal{C}_{N_{R}}^{N_{i}} P_{e}^{N_{i}}\left(1-P_{e}\right)^{N_{R}-N_{i}}$, where $\mathcal{C}$ is the binomial coefficient. The probability $P_{d}$ to dissolve any jam of size $N$ is the cumulative probability of 
all escape events with $N_{i} \geqslant N$ and $P_{d}=\Sigma_{i} \mathcal{B}\left(N_{i} \geqslant N\right) . P_{d}{ }^{-1}$ represents the duration of a jammed state period.

The average flux $\left(Q_{n, f} P_{j}^{-1}+Q_{n, c} P_{d}^{-1}\right) /\left(P_{j}^{-1}+P_{d}^{-1}\right)$ is presented by a solid line in Fig. 7 and shows a remarkable agreement with the simulations.

\section{Modification of the time distributions}

In the following, the rules of the lattice model are modified to show how the coexistence region can be shifted by a modification of the particle fluctuations but without modification of the average quantities defining the free and the congested regimes.

\section{Escape time distribution}

So far, the numerical solution of the lattice model has been proposed with an escape probability $P_{e}$ independent of the time step. In this case, the distribution $P(\tau)$ for an escape after a time $\tau$ is exponential with $P(\tau) \propto \exp \left(-P_{e} \tau\right)$. In addition to the exponential distribution for the escape time, a Gaussian distribution $P(\tau) \propto \exp \left[-\left(\tau-P_{e}^{-1}\right)^{2} / 2 \delta \tau^{2}\right]$ is tested. The distribution is centered on an escape time $P_{e}{ }^{-1}=$ 20 time steps with a standard deviation $\delta \tau=4$ time steps. This narrow distribution of escape times limits fluctuations in $n_{J}(t)$, making fluctuations down to $n_{J}(t)=0$ very unlikely, and thus it inhibits the dissolution of even a small jam. The simulation results are represented by open squares in Fig. 7 , and a narrow coexistence region is observed around $N=10$. For $N \geqslant 11$, the regime is fully in the congested branch. The extent of the coexistence region as well as the efficiency of the traffic are therefore highly sensitive to the distribution of escape times.

The numerical results of the lattice model are consistent with the results obtained in Fig. 5 if one considers that the escape time distribution is approximately exponential for the dry granular particles and Gaussian for the artificial fish.

\section{Headway time distribution}

A regulation rule is implemented in the simulations to mimic the effect of the damper on the distance regulation. In the experiments, the effect of the damper combines a slowing-down and a distance regulation of the particles. The slowing-down is known to increase the flux at high densities according to the "slower is faster" effect. In the lattice model, the regulation rule is imposed for the cell tagged $R$ in Fig. 7, where a particle can move only if the next $n=5$ cells are empty. Contrary to the experiments, this rule does not induce a velocity reduction for an isolated particle. The simulation results with open circles show an improvement of the traffic, and the coexistence region is extended up to $N=21$. In the range $15 \leqslant N \leqslant 18$, the flux is increased by a factor of 1.6.

\section{CONCLUSION}

A relation similar to the fundamental diagram in traffic engineering is reported for an ideal model of self-propelled elements in both experiments and a lattice model. The jams are triggered at a bottleneck of the track, and the traffic performances decrease well below the saturation of the whole track. A coexistence region is identified where the flow state sponta-

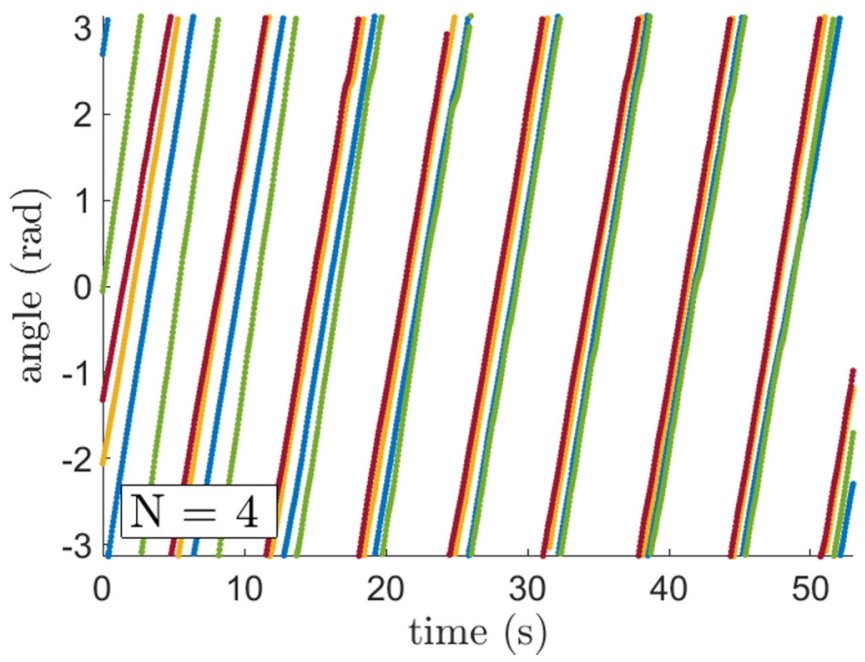

FIG. 8. Angular trajectories of $N=4$ particles. Each particle is represented by a single color.

neously switches between a free and a congested regime with a fixed number of particles in the track.

The role of the particle fluctuations is crucial to account for the existence of the coexistence region. The range of the coexistence region strongly depends on the escape time statistics from a jam and is very sensitive to the headway time distribution for the particles arriving at the bottleneck without a jam. The lattice model is used to show that the coexistence region may be shifted if the time or distance distributions between the particles are modified, even if the average traffic remains unchanged in the limits of the free and the congested regimes. The simulations are used to show that the flux increases if the separation between particles is controlled in the free-flowing region. On the other hand, the simulations demonstrate that a nearly constant escape time for the escape in the presence of a jam has the opposite effect and results in a decrease of the flow performance.

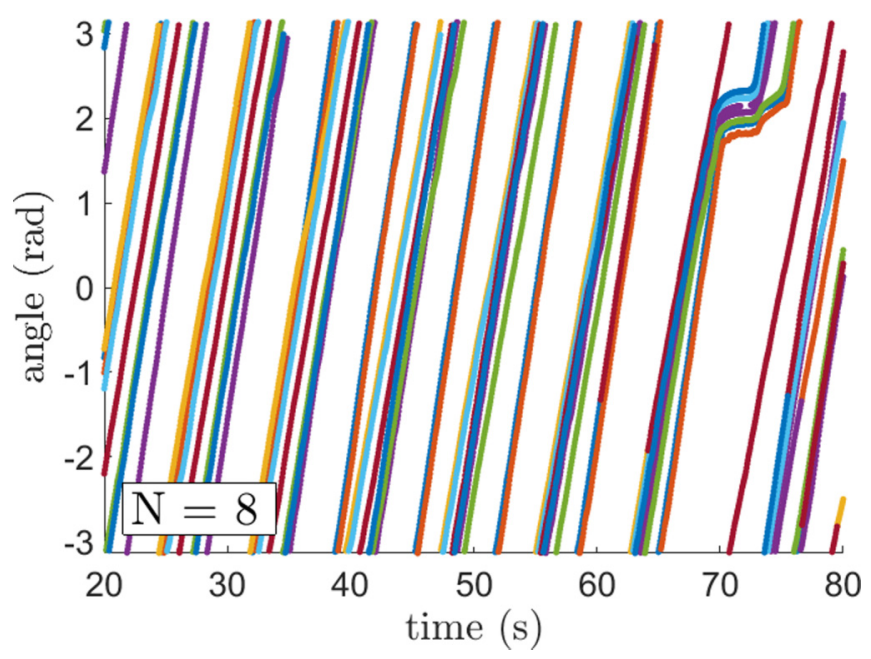

FIG. 9. Angular trajectories of $N=8$ particles. Each particle is represented by a single color. 


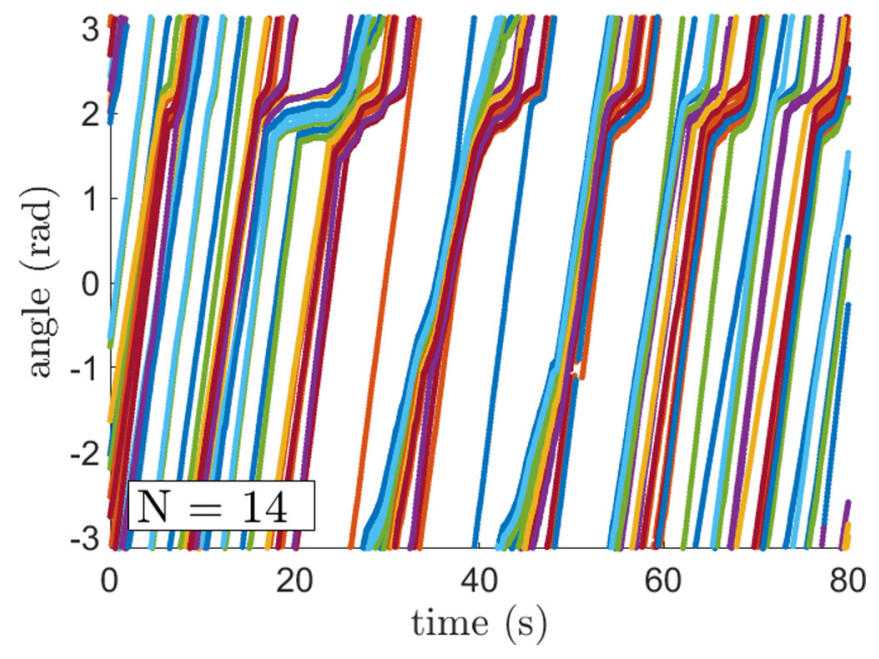

FIG. 10. Angular trajectories of $N=14$ particles. Seven colors are used to represent the trajectories of the 14 particles.

\section{ACKNOWLEDGMENTS}

J.S.L. acknowledges funding from IdEx (Initiative d'Excellence) Bordeaux Junior Chair.

\section{APPENDIX A: PARTICLE TRACKING AND FLUX MEASUREMENT}

The particle trajectories are extracted from image analysis. The recording camera has a frame rate of 20 frames per seconds. The image resolution is $962 \times 962$ pixels with RGB color in eight-bit depth. Each robot has a pair of colored stickers that are identified to obtain the location and the orientation of the particles. The angular position $\theta$ of the particles in the track is obtained from the 2D trajectories $\left\{X_{i}(t), Y_{i}(t)\right\}$ by $\theta=\arctan \left(Y_{i}(t) / X_{i}(t)\right)$.

The flux diagrams with $Q$ as a function of $N$ are obtained with a set of experiments where the number of particles in the track is fixed. For each particle number investigated $(N=2,3, \ldots)$, a sequence of $1-2 \mathrm{~min}$ in length is used to record the particle trajectories. The trajectories are processed to count the particles crossing the line $\theta=-90^{\circ}$. The flux $Q$ $\left(\mathrm{s}^{-1}\right)$ is obtained by dividing the counted particles $N_{\text {count }}$ by the duration $T_{\text {seq }}$ of the sequence. A visual inspection of the images is used to add the contribution of the particles missed by the algorithm.

\section{APPENDIX B: JAM SCENARIO}

The angular trajectories of the particles are presented in Figs. 8, 9, 10, and 11. The slope of the trajectories is the

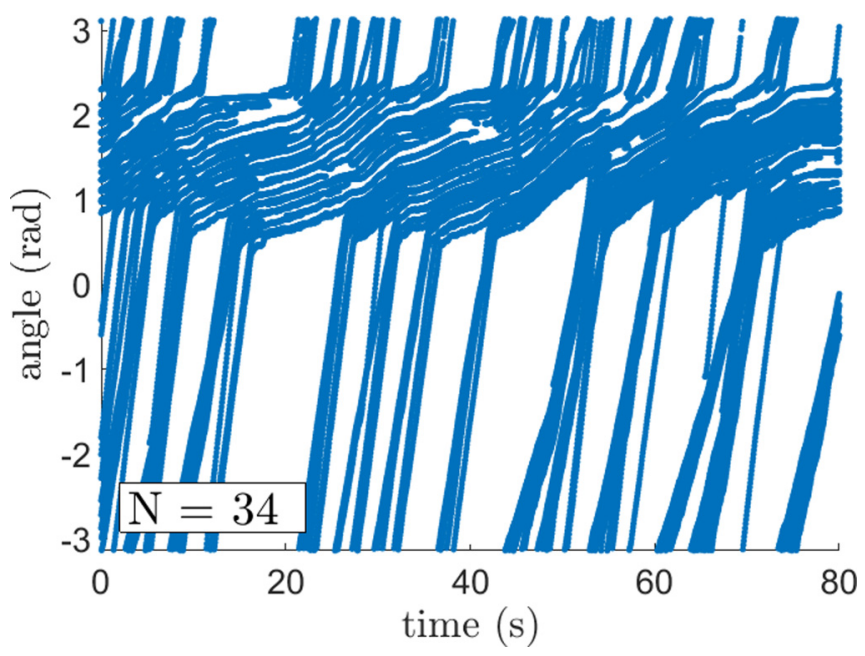

FIG. 11. Angular trajectories of $N=34$ particles.

angular velocity of the particles with respect to the center of the track.

For $N=4$ (Fig. 8), a free traffic regime is observed. The particles have a homogeneous velocity in the track. The formation of a moving cluster is observed between 0 and $40 \mathrm{~s}$ because a slightly slower particle is slowing down the other following particles. Even if the double lane sector allows overtaking, the overtaking by the fastest particles is not systematically observed because of the random dynamics of the particles in the track. An overtaking event is occurring at $49 \mathrm{~s}$ and then a spreading of the cluster is observed.

For $N=8$ (Fig. 9), the traffic is still dominantly free but very short blocking events can be observed. A jam with a duration of $5 \mathrm{~s}$ is observed around $70 \mathrm{~s}$. A clustering of the particles acting as a precursor of the jam event is observed between $60 \mathrm{~s}$ and the blocking event at $70 \mathrm{~s}$. Because there are not enough particles to maintain the jam since some particles are still escaping from the front of the jam, the jam is rapidly dissolved. The dissolution of the jam results in a spreading of the cluster at times greater than $70 \mathrm{~s}$.

For $N=14$ (Fig. 10), the traffic is switching between jammed and free states. There are enough particles in the track to observe multiple blocking events. However, the number of particles is not sufficient to maintain enough particles in the jam, and the intrinsic fluctuations of the jam size are reaching $n_{J}(t)=0$ after a short time, typically less than $10 \mathrm{~s}$.

For $N=34$ (Fig. 11), there are enough particles in the track to maintain a persistent jam. The jam is slowly flowing and the particles escape with a time distribution $P\left(T_{e}\right)$.
[1] V. Narayan, S. Ramaswamy, and N. Menon, Science 317, 105 (2007).

[2] J. Deseigne, O. Dauchot, and H. Chaté, Phys. Rev. Lett. 105, 098001 (2010).

[3] A. Bricard, J.-B. Caussin, N. Desreumaux, O. Dauchot, and D. Bartolo, Nature (London) 503, 95 (2013).
[4] E. Bonabeau, M. Dorigo, and G. Théraulaz, Swarm Intelligence: From Natural to Artificial Systems (Oxford University Press, Oxford, 1999), p. 1.

[5] B. S. Kerner, Phys. World 12, 25 (1999).

[6] D. Chowdhury, L. Santen, and A. Schadschneider, Phys. Rep. 329, 199 (2000). 
[7] D. Helbing, Rev. Mod. Phys. 73, 1067 (2001).

[8] M. Takayasu, H. Takayasu, and K. Fukuda, Physica A 277, 248 (2000).

[9] D. Helbing, I. Farkas, and T. Vicsek, Nature (London) 407, 487 (2000).

[10] E. Altshuler, O. Ramos, Y. Núñez, J. Fernández, A. BatistaLeyva, and C. Noda, Am. Nat. 166, 643 (2005).

[11] D. Helbing, L. Buzna, A. Johansson, and T. Werner, Transport. Sci. 39, 1 (2005).

[12] A. J. Liu and S. R. Nagel, Nature (London) 396, 21 (1998).

[13] T. S. Majmudar, M. Sperl, S. Luding, and R. P. Behringer, Phys. Rev. Lett. 98, 058001 (2007).

[14] A. Janda, D. Maza, A. Garcimartín, E. Kolb, J. Lanuza, and E. Clément, Europhys. Lett. 87, 24002 (2009).

[15] I. Zuriguel, A. Janda, A. Garcimartín, C. Lozano, R. Arévalo, and D. Maza, Phys. Rev. Lett. 107, 278001 (2011).

[16] M. J. Lighthill and G. B. Whitham, Proc. R. Soc. London, Ser. A 229, 317 (1955).

[17] S. P. Hoogendoorn and P. H. Bovy, Proc. Inst. Mech. Eng., Pt. I: J. Syst. Contr. Eng. 215, 283 (2001).

[18] K. Nagel and M. Schreckenberg, J. Phys. I 2, 2221 (1992).

[19] S. Maerivoet and B. De Moor, Phys. Rep. 419, 1 (2005).

[20] K. Nishinari, K. Sugawara, T. Kazama, A. Schadschneider, and D. Chowdhury, Physica A 372, 132 (2006).

[21] N. J. Suematsu, S. Nakata, A. Awazu, and H. Nishimori, Phys. Rev. E 81, 056210 (2010).

[22] B. Tian, W.-P. Sun, M. Li, R. Jiang, and M.-B. Hu, Chin. Phys. B 27, 038902 (2018).

[23] G. A. Patterson, P. I. Fierens, F. Sangiuliano Jimka, P. G. König, A. Garcimartín, I. Zuriguel, L. A. Pugnaloni, and D. R. Parisi, Phys. Rev. Lett. 119, 248301 (2017).

[24] B. S. Kerner, Phys. Rev. Lett. 81, 3797 (1998).

[25] 75 Years of the Fundamental Diagram for Traffic Flow Theory: Greenshields Symposium: 2008, Woods Hole, Massachusetts, Transportation Research Circular (Transportation Research Board, 2011), URL https://books.google.fr/books?id= x8s4ngAACAAJ.
[26] A. Seyfried, B. Steffen, W. Klingsch, and M. Boltes, J. Stat. Mech.: Theor. Exp. (2005) P10002.

[27] S. Kurata and T. Nagatani, Physica A 318, 537 (2003).

[28] K. Nassab, M. Schreckenberg, A. Boulmakoul, and S. Ouaskit, Physica A 369, 841 (2006).

[29] L. Davis, Physica A 451, 320 (2016).

[30] T. Nagatani, J. Phys. A 26, 6625 (1993).

[31] Y. Sugiyama, M. Fukui, M. Kikuchi, K. Hasebe, A. Nakayama, K. Nishinari, S.-i. Tadaki, and S. Yukawa, New J. Phys. 10, 033001 (2008).

[32] A. Kesting, M. Treiber, and D. Helbing, Transport. Res. Rec. 1999, 86 (2007).

[33] S. A. Soria, R. Josens, and D. R. Parisi, Safety Sci. 50, 1584 (2012).

[34] A. Garcimartín, I. Zuriguel, J. Pastor, C. Martín-Gómez, and D. Parisi, Transport. Res. Proc. 2, 760 (2014).

[35] C. Gershenson and D. Helbing, Complexity 21, 9 (2015).

[36] J. M. Pastor, A. Garcimartín, P. A. Gago, J. P. Peralta, C. MartínGómez, L. M. Ferrer, D. Maza, D. R. Parisi, L. A. Pugnaloni, and I. Zuriguel, Phys. Rev. E 92, 062817 (2015).

[37] P. Lin, J. Ma, T. Liu, T. Ran, Y. Si, and T. Li, Physica A 452, 157 (2016).

[38] F. Soriguera, I. Martínez, M. Sala, and M. Menéndez, Transport. Res. Pt. C: Emerging Technol. 77, 257 (2017).

[39] A. DeSimone and A. Tatone, Eur. Phys. J. E 35, 85 (2012).

[40] L. Giomi, N. Hawley-Weld, and L. Mahadevan, Proc. R. Soc. A 469, 20120637 (2013).

[41] A. Deblais, T. Barois, T. Guerin, P.-H. Delville, R. Vaudaine, J. S. Lintuvuori, J.-F. Boudet, J.-C. Baret, and H. Kellay, Phys. Rev. Lett. 120, 188002 (2018).

[42] H. H. Wensink and H. Löwen, Phys. Rev. E 78, 031409 (2008).

[43] N. Tarcai, C. Virágh, D. Ábel, M. Nagy, P. L. Várkonyi, G. Vásárhelyi, and T. Vicsek, J. Stat. Mech.: Theor. Exp. (2011) P04010.

[44] R. Sánchez and P. Díaz-Leyva, Physica A 499, 11 (2018).

[45] See Supplemental Material at http://link.aps.org/supplemental/ 10.1103/PhysRevE.99.052605 for a set of movies recorded for a varying number $N$ of particles in the track. 